\title{
Genetic Screening Offers New Hope for Prostate Cancer Patients
}

\author{
Kathryn Leake* \\ Oncology Solutions, USA
}

*Corresponding author: Kathryn Leake, Director, Oncology Solutions, Houston, Texas, USA.

Received Date: July 10, 2019

Published Date: July 16, 2019

\section{Mini Review}

The good news for prostate cancer patients currently undergoing chemotherapy treatments is that, genetic markers which once seemed the kiss of death may now permit classification into a group of new prostate cancer treatments! Hospitals should be made aware that if their prostate cancer patients are PTEN or p53 mutant, these patients may benefit from therapy blocking interleukin-6 (IL6)[1]. With our present-day individualized approach to medicine, standard diagnostic tests can be employed, such as a cheek swab, to flag patients who are candidates for this novel treatment. This novel immunotherapy is presently in drug development and will treat metastasis in the most refractory prostate cancer patients. What does this novel IL6-based therapy mean for a prostate cancer patient who is nearing Stage T3 or T4 (TNM, Tumor/Nodes/Metastasis system [2]) or has already entered it? It means a fighting chance.

Interleukin- 6 based immunotherapy should also benefit prostate cancer patients who are nonresponders to androgen ablation therapy. Until now, these patients were left with little hope. While androgen ablation therapy is still the best treatment of choice for prostate cancer patients, it is still a mystery why some patients do not respond well to it. Thanks to Lloyd Trotman of Cold Spring Harbor and his postdoctoral fellow, Dawid Nowak, of Weill Cornell Medicine, who is first author on the paper, [1] things have changed. They developed a murine model of prostate cancer metastasis, called RapidCap. RapidCap are genetically engineered mice undergoing metastasis of prostate cancer. Interestingly, most cell lines of prostate cancer for study in the laboratory, are transformed by genetic loss of PTEN [1]. Cell lines PC-3 and LNCaP are modified in this way. How do IL6 and PTEN affect prostate cancer metastasis? Interleukin 6 has been associated with STAT protein activation for years. However, we now understand through watching prostate cancer metastasis in Trotman and Nowak's murine model, that signaling is redirected to IL6, STAT and MYC pathways of cellular proliferation when a prostate cancer patient expresses haploinsufficiency for PTEN and p53 [3]. Another drug target Nowak and Trotman identified is the phosphatase PHLPP2. Inhibiting PHLPP2's phosphatase activity on Threonine 58 of MYC, a well-known oncogene in many types of cancer, switches it's signaling from tumor progression towards destabilization and tumor suppression [3].

While the aforementioned studies are honing in on the etiology of prostate cancer metastasis, other researchers have been working to develop novel therapeutic strategies that are not associated with etiology. For example, the non-ABC-type transporter, RLIP76, was identified in 2009 as an excellent drug target in the fight against drug-resistance of prostate cancer to present chemotherapies. Still, translational studies have not been funded. This is unacceptable, as RLIP76 has proven to be elevated in PC-3 cells such that blocking antibodies administered to murine models cause a log-fold reduction in tumor xenografts. Singhal et al also showed that RLIP76 protein and activity are greater in cancer cells compared to normal controls [4]. So, prostate cancer patients can hold out hope that even if their disease is hormone-therapy resistant, immunotherapy via an IL6 blocking antibody, or a PHLPP2 inhibitor should slow their disease progression. And, finally, once RLIP76 blocking antibodies are investigated in clinical trials, even prostate cancer's drug resistance will be defeated.

\section{Acknowledgment}

None.

\section{Conflict of Interest}

No conflict of interest.

\section{References}

1. Cancer Discovery (2015).

2. BMJ Group (2009) Prostate cancer: How far has your cancer spread? The TNM system.

3. J Cell Biology (2019).

4. Biochem Pharmacol (2009). 